Older People during the lockdown period in 2020 was summarised in two case scenarios. One scenario represents cases with known background of dementia, while the other scenario represents new referrals during the lockdown period with no known psychiatric background. In those cases, the visual hallucinations started during lockdown with no clear cause, did not respond to psychotropic medications, physical health investigations were all normal and hallucinations improved markedly with the end of the lockdown and social isolation.

Discussion. From clinical practice point of view, during the period of lockdown in the COVID-19 pandemic, visual hallucinations has been one of the commonest presentations reported to the Community Mental Health Teams for Older People. Families were calling frequently reporting that their loved ones were "seeing things". Possible underlying causes include: social isolation, sensory and perceptual deprivation, visual impairment and Charles Bonnet syndrome, lack of cognitive stimulation activities with progress of dementia, superimposed delirium, in addition to depression secondary to loneliness, reduction in community support, increased alcohol consumption and negative effects of repeated media consumption.

Conclusion. There has been a marked increase in reporting visual hallucinations in the shielding older people population in the community during the period of lockdown in the COVID-19 pandemic. This shielded population was not exposed to COVID-19, so it didn't give an explanation to this new phenomenon. Though there are multiple possible causative factors, the effect of the lockdown itself with its resultant social isolation and sensory deprivation remains to be the most significant. Shielding the older people population throughout the COVID-19 pandemic came as an essential measure as the physical safety and preventing loss of lives was the priority; however the lockdown had significant negative effects on the mental health of the shielding population. It remains unclear if those negative effects are going to be reversible in the future, resulting in poor quality of life.

\section{Hypnic headache: a rare type of primary headache disorder}

Muhammad Sayed Inam ${ }^{1 \star}$, Saifun Nahar ${ }^{2}$ and Mohammad Zubayer Miah $^{3}$

${ }^{1}$ Sylhet MAG Osmani Medical College and Hospital; ${ }^{2}$ National Institute of Mental Health Dhaka and ${ }^{3}$ Shaheed Tajuddin Ahmed Medical College

${ }^{*}$ Corresponding author.

\section{doi: 10.1192/bjo.2021.349}

Objective. Hypnic Headache are is a very rare primary headaches that affect the elderly, with an average age of 60 years. Research in the areas of neurophysiology and treatment options for Hypnic Headache are necessary in order to better understand, and improve outcomes for this rare headache disorder.

Case report. Mr. X is a 70-year-old patient, has been presenting with the complaints of headache during sleep at night for the last 1 year. The Headache started after 3 to 4 hours after falling asleep. Due to headache, he wakes up from sleep around 03:00 to 04:00 am almost every night and his headache persist for 30 to 40 minutes. After waking up from sleep he keeps himself busy with religious activity and the headache gradually resolves. He then goes back to bed again.

Mr. X also informed that, the headache is dull in nature and located in left temporo occipital region. It is not associated with photophobia, phonophobia, nausea, vomiting, tearing or discomfort in the leg. He gives no history of early morning headache or day time headache, sleep disorder, snoring or sleep apnea. He has no past history of trauma to the head, fainting attack, unconsciousness, weakness or paralysis of limbs, seizures or non-epileptic seizures. He is an nonsmoker, non-alcoholic, non-hypertensive \& non-diabetic person.

On general examination, his heart rate is 70 beats/min, blood pressure 138/68 mm of Hg. There are no anemia, jaundice or oedema present in him. His both lung fields are clear. On neurological examinations there are nothing abnormality detected. His Serological investigations, CBC (Complete Blood Count) FBS (Fasting Blood Glucose), lipid profile are within normal limit. CT scan of the brain is normal. There are no cerebral atrophy or volume loss compatible with age.

Mr. X was treated by several general practitioners with paracetamol, diclofenac sodium, mefenamic acid, tramadol hydrochloride. He used these drugs either singly or in combinations. But with this treatment there were no significant improvement occurs. Mr. X is scared and depressed for his sleep time headache.

Discussion. Hypnic headache is a very rare headache disorder. It occurs in age groups over 60 years. It is occur at night during in sleep and waking the patient up, hence the name of it "alarm clock headache". It is commonly unilateral and lasts for 15 minutes to 4 hours. Hypnic headache commonly dull or throbbing in character and does not make the patient restless, unlike in Cluster Headache. After waking up from sleep, most patients engage in some activity. Hypnic headache is not associated with rhinorrhea, tearing and ptosis. Diagnosis is mainly clinical. Secondary causes headache must be excluded. International Classification of Headache Disorders 3rd Edition (ICHD-3)-beta provides diagnostic criteria for hypnic headache. Pathophysiology of hypnic headache is not clearly identified. Usual treatment options of Hypnic headache includes bed time coffee, lithium carbonate, indomethacin. Our patient fulfil all the criteria of Hypnic headache and he fells improvement with Indomethacin $50 \mathrm{mg}$ in devided doses.

Conclusion. Hypnic Headache is a very rare type of primary headache. It should be diagnosed only after other secondary causes of headache have been excluded. Caffeine, lithium carbonate, flunarizine, indomethacin, used to treat the patient of Hypnic Headache. Lack of study and awareness about these disorders can lead to delays in diagnosis and treatment. Clinical trials are needed to find out proper treatment, but it will be difficult to perform because of the rareness of this disorder.

\section{The impact of the COVID-19 pandemic on obsessive compulsive disorder: a single case study}

Josephine Sibanda-Mbanga ${ }^{\star}$ and Anusha Govender

South West London \& St George's Mental Health NHS Trust ${ }^{*}$ Corresponding author.

doi: 10.1192/bjo.2021.350

Objective. A growing body of research evidence shows that individuals with Obsessive Compulsive Disorder (OCD) have been negatively affected by the COVID-19 pandemic including deterioration of OCD symptoms plus relapse from previously well-controlled OCD. The impact of the COVID-19 pandemic is discussed in a single case study of a patient with OCD consisting of contamination concerns. In addition, the effectiveness of providing Exposure and Response Prevention (ERP) virtually is evaluated with regards to the treatment outcome in COVID-19 related OCD.

Case report. This study describes a 41-year-old, single, employed female with OCD consisting of concerns of contamination and infecting others thereby causing harm. The total duration of the disorder is 32 years with the most recent presentation being of three years duration. Relapse during the pandemic resulted in 
OCD symptoms being solely concerned with COVID-related contamination. The questionnaires routinely completed at the time of assessment and treatment were the Obsessive Compulsive Inventory (OCI); Yale Brown Obsessive Compulsive Scale (YBOCS); Beck Depression Inventory (BDI). Clinical data were collated and analysed prior to and during the pandemic. Treatment consisted of ERP and was adapted for provision via a virtual platform. ERP involved exposure to a graded hierarchy of COVID-specific anxiety-provoking situations modified to take government guidelines into consideration.

Discussion. Prior to the COVID-19 pandemic the patient's response to treatment with cognitive behavioural therapy (CBT) including ERP indicated a $79 \%$ improvement in OCD symptoms on self -rated measures. The impact of the pandemic led to a significant $65 \%$ deterioration in OCD symptoms, regarding COVID-19 contamination concerns. Intervention with ERP resulted in $73 \%$ improvement over a three-month period. Measures of depression symptoms indicated an $80 \%$ improvement pre-COVID, with a $78 \%$ deterioration at relapse. Following treatment, the patient also showed a $65 \%$ improvement in depression symptoms. Improvements have been maintained at one month follow-up.

Conclusion. The case study supports literature indicating the exacerbation of OCD symptoms due to the COVID-19 pandemic for patients with contamination fears and washing compulsions. The promising results support the use of ERP as an effective treatment for COVID-related OCD symptoms. It also validates the provision of CBT interventions virtually to ensure accessibility of treatment to OCD sufferers.

\section{A brief novel intervention for acrophobia (fear of heights)}

\section{E. Naomi Smith*}

South London and Maudsley Training Scheme

${ }^{*}$ Corresponding author.

doi: 10.1192/bjo.2021.351

Objective. To investigate a unique brief intervention, which offers a combination of neuro-linguistic programming and practical graded exposure therapy, to overcome a fear of heights.

Background. A fear of heights or acrophobia is common and often deters people from perusing activities like climbing. It can also interfere with routine activities of daily living.

Case report. This two-day intervention is set in the Peak District (Derbyshire, UK) and works with a maximum of eight individuals to four instructors. The first half-day involves working with a psychotherapist using neurolinguistic programing techniques. The next 1.5 days involves graded exposure using abseiling over gradually increasing heights, to a final height of approximately 40 feet. Discussion. All eight individuals on the two-day course felt their fear of highs had significantly decreased. All eight individuals would recommend this intervention to others suffering from a fear of heights.

Conclusion. It is noteworthy that the group undergoing this intervention were self-selected and highly motivated to overcome their fear of heights. The sample size was small and outcome measures were subjective. However, this is a novel and effective approach to helping people overcome their fear of heights. Further research with larger sample sizes would be beneficial in further assessing the impact of this intervention.

Declaration: Permission was granted by the organizers of this intervention to submit an abstract to conference. There are no conflicts of interests. This intervention is run by a private company 'Will4Adventure', I have no finical or other interests in this company. I privately funded my own place on this course.

\section{Access to firearms: essential factor for risk management in psychiatry}

Donnchadh Walsh ${ }^{1 *}$, Una Fallon ${ }^{2}$, Sonn Patel ${ }^{3}$ and Elizabeth Walsh ${ }^{3}$

${ }^{1}$ School of Medicine, University College Dublin; ${ }^{2}$ Galway Menal Health Service and ${ }^{3}$ University Hospital Galway

${ }^{\star}$ Corresponding author.

doi: 10.1192/bjo.2021.352

Objective. Increase awareness of the risk associated with access to firearms in clinical practice.

Case report. A 57-year-old man, with a 30 year history of schizophrenia, was reviewed routinely at home. His illness is predominantly characterised by chronic delusions of a grandiose nature. He believes he has been offered various senior employment positions and has acted on these beliefs by presenting at workplaces in business attire. He has no insight into his condition. At review, he described awakening a week earlier in a panic and seizing hold of his legally held shotgun. He planned to shoot out the window as he believed people were breaking in. His wife prevented him from doing so by taking the gun and hiding it. A few days later he found the gun and intended to frighten off potential pursuers by pretending to shoot birds. He was persuaded to surrender the gun and it was taken to the local Garda (Police). A short time later, he presented to Garda Headquarters, over an hour away, seeking the return of his gun. At review, he had limited insight into the potential seriousness of the situation. The team immediately liaised with Gardaí, a HCR- 20 risk assessment was completed and clozapine levels checked.

Discussion. We had not know that our patient owned a shotgun despite very regular contact with him. During a comprehensive psychiatric history we routinely ask about risk of harm to self and others, but rarely ask specifically about access to or ownership of guns. Working on a farm, rural living or having an interest in shooting sports may raise the issue. Suicide, security breaches and homicide are the main risks conferred by firearms in mental illness. Mental illness is not necessarily prohibitive to gun ownership. Applicants for gun certificates in the UK must disclose specific medical conditions, including a psychotic illness, and an automatic medical report is sought. In the Irish Republic it is the responsibility of the applicant to declare any specific physical or mental health condition. Although a medical report may be sought, it is not automatic in all cases. Lack of insight into psychotic illness may potentially influence self-declaration upon application for a certificate.

Conclusion. Awareness of a persons access to firearms should be part of our routine risk assessment.

\section{Michael Kohlhaas Syndrome - Taking the court to court}

Louisa Ward*

Worcestershire Health and Care NHS trust ${ }^{*}$ Corresponding author.

doi: 10.1192/bjo.2021.353

Objective. To explore Michael Kohlhaas syndrome and development of litigious paranoia. 